\title{
Biomimetic Design of Artificial Micro-vasculatures for Tissue Engineering
}

\author{
Robert W. Barber and David R. Emerson
}

Science and Technology Facilities Council, Daresbury Laboratory, Warrington, UK

\begin{abstract}
Summary - Over the last decade, highly innovative micro-fabrication techniques have been developed that are set to revolutionise the biomedical industry. Fabrication processes, such as photolithography, wet and dry etching, moulding, embossing and lamination, have been developed for a range of biocompatible and biodegradable polymeric materials. One area where these fabrication techniques could play a significant role is in the development of artificial micro-vasculatures for the creation of tissue samples for drug screening and clinical applications. Despite the enormous technological advances in the field of tissue engineering, one of the major challenges is the creation of miniaturised fluid distribution networks to transport nutrients and waste products, in order to sustain the viability of the culture. In recent years, there has been considerable interest in the development of microfluidic manifolds that mimic the hierarchical vascular and parenchymal networks found in nature. This article provides an overview of microfluidic tissue constructs, and also reviews the hydrodynamic scaling laws that underpin the fluid mechanics of vascular systems. It shows how Murray's law, which governs the optimum ratio between the diameters of the parent and daughter branches in biological networks, can be used to design the microfluidic channels in artificial vasculatures. It is shown that it is possible to introduce precise control over the shear stress or residence time in a hierarchical network, in order to aid cell adhesion and enhance the diffusion of nutrients and waste products. Finally, the paper describes the hydrodynamic extensions that are necessary in order to apply Murray's law to the rectangular channels that are often employed in artificial micro-vasculatures.
\end{abstract}

Key words: biomimetics, lab-on-a-chip, microfluidics, micro-vasculatures, Murray's law, tissue engineering.

\begin{abstract}
Address for correspondence: Robert W. Barber, Centre for Microfluidics and Microsystems Modelling, STFC Daresbury Laboratory, Warrington, Cheshire WA4 4AD, UK.

E-mail: robert.barber@stfc.ac.uk
\end{abstract}

\section{Introduction}

Over the last two decades, significant advances have been made in the field of tissue engineering (1-14), but despite many enormous achievements, various barriers prevent the widespread use of tissue-engineered products. These barriers include regulatory and ethical issues, as well as many scientific and engineering challenges. As detailed by $\mathrm{Du}$ et al. (15), the challenges include questions related to the harvesting and acquisition of cells, and problems associated with the creation of artificial tissues that have the same biomechanical and metabolic characteristics as natural tissues. However, as discussed by Borenstein et al. (7), the most important challenge to the development of in vitro tissue cultures appears to be the difficulty in creating suitable vascularised structures that mimic the intricate capillary networks found in nature. In the absence of a suitable capillary network, the thickness of any engineered tissue is generally restricted to $150-200 \mu \mathrm{m}$, due to the limited distance that oxygen and other nutrients can diffuse before being consumed by the cells (16).

The advent of micro-fabrication technologies and, in particular, the development of microfluidic systems, has provided new opportunities to create miniature vascularised structures that can be used in tissue-engineering applications. Current microfabrication technologies are able to achieve resolutions down to $0.1 \mu \mathrm{m}$, and can pattern surfaces and create intricate networks of channels over large areas of substrate. One of the most popular microfabrication technologies is soft lithography (17), which employs poly(dimethylsiloxane) (PDMS) or fluorosilicone elastomers to fabricate microstamps and micro-moulds. Although the original master has to be fabricated via conventional photolithography, once it is created, the master can be reused many times without the need for cleanroom facilities, which makes the technique ideal for mass-production. In addition, micro-fabrication processes have been developed for a range of biocompatible and biodegradable polymeric materials, such as poly(lactic-co-glycolic acid) (PLGA; 18 ), poly(glycerol sebacate) (PGS; 19) and hydrogels (20).

One of the most exciting long-term goals for tissue engineering is to provide replacement organs for patients with end-stage organ failure (16). Whilst significant progress has already been made in developing micro-fabricated renal-assist and renal-replacement devices (21), the ultimate goal of creating complete replacement organs is possibly many decades away. Another very promising application area for tissue engineering is in the 
development of surrogate in vivo cell-based lab-ona-chip devices that mimic the complex physiological responses found in mammals. Sometimes referred to as 'animal-on-a-chip' devices $(22,23)$, these micro-fabricated systems are composed of an arrangement of interconnected compartments that each contain a culture of cells and mimic the primary organs of a living animal. Microfluidic channels connecting the various compartments permit the recirculation of a culture medium, in an analogous manner to the blood supply in an animal. Proof-of-principle experiments have shown that animal-on-a-chip devices can replicate the complex inter-organ or inter-tissue interactions found in living animals, and are able to mimic the immune response to toxic or therapeutic compounds (24). Such systems could be used to bridge the gap between traditional cell-based assays and animal studies, and could have a significant impact in reducing, and possibly eliminating, many forms of animal testing.

\section{Vascularised Tissue Constructs}

The pioneering work on artificial vasculatures generally employed planar or two-dimensional (2-D) micro-fabrication technologies. For example, Kaihara et al. (5) used conventional photolithographic techniques to generate a pattern of bifurcating channels on silicon or Pyrex wafers. They demonstrated that endothelial cells and hepatocytes could be cultured on the substrate, and subsequently lifted from the pattern as a single-cell monolayer. Later, Borenstein et al. (7) used soft lithographic techniques to create a moulded 2-D PDMS substrate that was bonded to a second PDMS layer to create a network of enclosed microchannels. One of the practical limitations of using silicon, Pyrex or PDMS as substrates for tissue engineering is that the materials are not biodegradable and have limited biocompatibility. To address this limitation, King et al. (18) investigated the potential of biodegradable scaffolds manufactured from PLGA. The micro-vasculatures were created from a PLGA membrane, and were laminated by thermal bonding to create the enclosed micro-channels. Although PLGA-based microfluidic systems offer the possibility of a very versatile and inexpensive fabrication route, there are potential concerns about the inflammatory immune response to the material. In addition, PLGA scaffolds are very brittle and lack the elasticity of natural tissues. To overcome some of these limitations, Wang et al. (25) and Bettinger et al. (19) have investigated the use of PGS, a biocompatible elastomer that is already approved for use in medical applications. PGS substrates can be patterned by using a silicon master, then bonded to a flat film to create the vascular networks. A num- ber of studies have indicated that three-dimensional (3-D) tissue cultures behave quite differently from 2-D cultures. Weaver et al. (26) have shown that 3-D cultures of breast cancer cells can become non-cancerous in the presence of antibodies for specific integrin receptors; unfortunately, this effect cannot be replicated in conventional 2-D tissue cultures. It is clear that cells respond to local stimuli, such as cell-to-cell contact and mechanical stresses imposed by the environment, and these effects are unlikely to be replicated within a 2-D environment. The creation of $3-\mathrm{D}$ microfluidic scaffolds is therefore of paramount importance for tissue engineering applications, since 2-D constructs are unable to mimic the precise in vivo cellular conditions. Unfortunately, whilst conventional photolithographic and soft-lithographic techniques are able to create $2-\mathrm{D}$ vascularised tissue scaffolds, the construction of $3-\mathrm{D}$ networks of channels remains a formidable challenge.

The most popular method of constructing 3-D scaffolds is to stack $2-\mathrm{D}$ vascularised systems together in a sandwich-like structure. For instance, Bettinger et al. (19) created a fully 3-D micro-fabricated scaffold by stacking a series of patterned PGS layers together. Other approaches for creating 3-D scaffolds involve the use of directwrite fabrication techniques. Such an approach was used by Therriault et al. (27), who constructed a 16-layer scaffold by depositing and exposing an organic ink layer-by-layer to build up a 3-D microvascular network. In addition, Lim et al. (28) have demonstrated a novel maskless direct-write method, by using an Nd:Yag (neodymium-doped yttrium aluminium garnet) laser to create multiple-depth channels by varying the power of the laser used to create the micro-channels. Figure 1 illustrates typical examples of micro-fabricated 3$\mathrm{D}$ vasculatures that have been developed for tissue-engineering applications.

\section{Biomimetic Analysis of Vascularised Networks}

Nature has always been an abundant source of inspiration, and, from the earliest of times, Man has sought to replicate ideas that have evolved naturally in plants and animals. Understanding these 'natural' optimal design strategies has led to an entirely new field of research, known as biomimetics. However, despite significant progress in the broader field of biomimetics, there appears to have been very few applications in the field of microfluidics.

One of the most important challenges in growing viable in vitro tissue cultures is the requirement to create an effective micro-circulation system for the distribution of nutrients and oxygen. It is clear that the dimensions of the micro-channels should be opti- 
Figure 1: Examples of bifurcating microvasculatures

a)

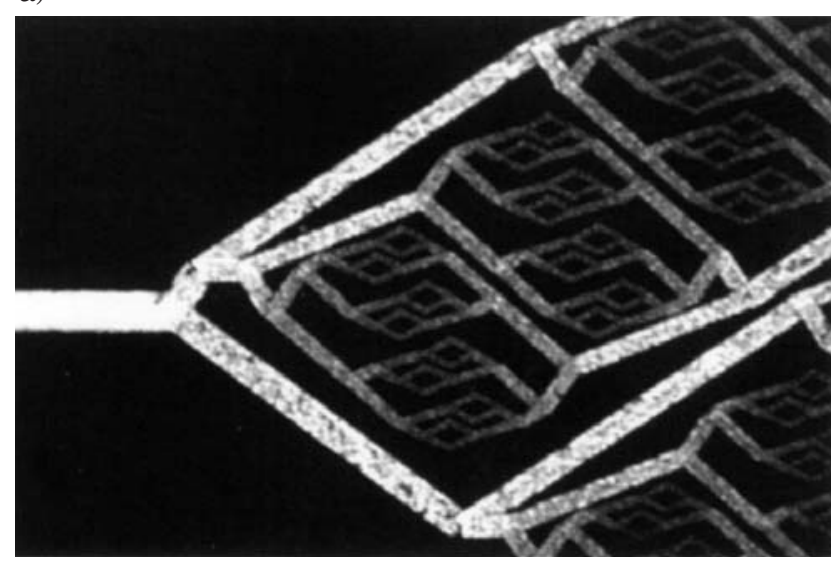

b)

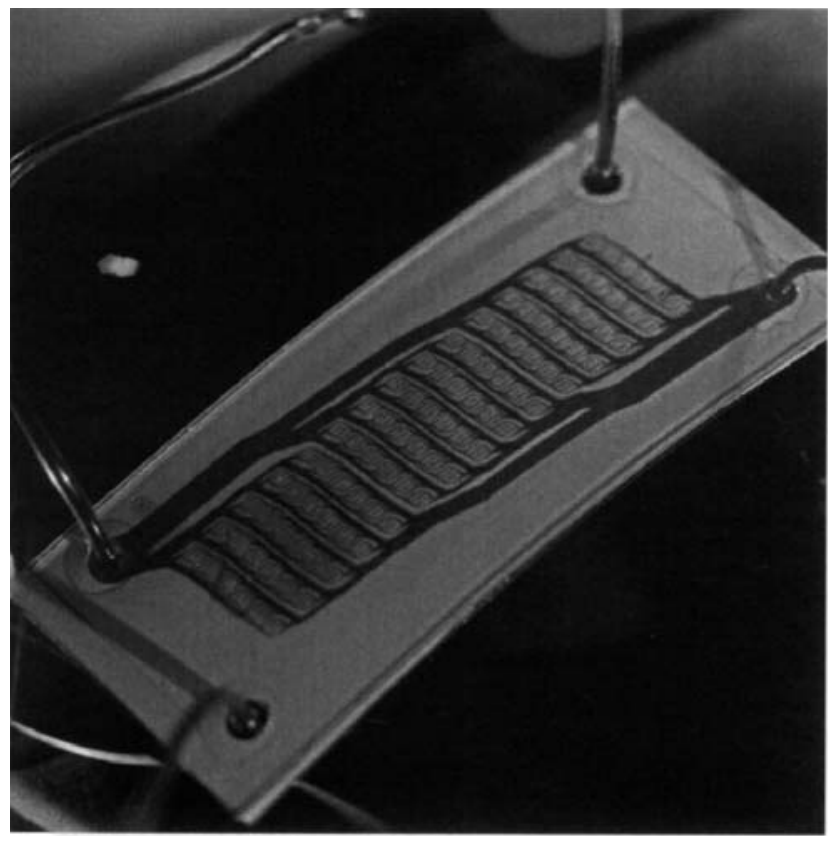

The figure shows typical examples of micro-fabricated manifolds developed for tissue-engineering applications. a) Eight generation multi-width, multidepth micro-vasculature fabricated by Lim et al. (28) by using a direct-write method. The fluorescent image clearly shows the difference in intensity levels corresponding to the varying channel depths.

Reproduced with the permission of The Royal Society of Chemistry (28).

b) A hepatocyte culture device developed by Borenstein et al. (12). The device consists of two layers: a microvascular network for blood flow and oxygenation, separated from a chamber for hepatocyte culture by a nanoporous membrane. Reproduced with the permission of Tissue Engineering (12). mised, so that the flow rates through the various pathways are uniform, and the total flow resistance of the network is compatible with the applied operating pressures. Although mathematical models are available for describing the branching properties of a vascular system, such techniques are rarely applied when designing artificial tissue constructs. The following sections show how the biomimetic scaling laws that govern biological distribution networks, can be used to design the optimal channel dimensions in artificial vasculatures.

\section{Theoretical Basis of the Hess-Murray Law}

One of the most distinctive features of biological distribution systems is their hierarchical structure and the successive division of vessels, which become smaller, both in length and diameter, as the network progresses (see Figure 2). Mathematical modelling of the cardiovascular system can be traced back to 1926, when Murray (29) used the principle of minimum work to derive a relationship between the diameter of the parent vessel and the optimum diameters of the daughter vessels in a branching network. The relationship is now known as Murray's law, which states that the cube of the diameter of the parent vessel equals the sum of the cubes of the diameters of the daughter vessels. However, Murray's law is actually a refinement of previous pioneering work by Hess (30), who used similar arguments and independently arrived at the same conclusion that the diameters obey a third-power rule. The earlier work of Hess has been noted by Weibel (31), Hahn et al. (32), and Bejan \& Lorente (33), and it is therefore probably more appropriate to refer to the rule as the Hess-Murray law. Unfortunately, the work of both Hess and Murray was overlooked for many years (34), but vascular trees and scaling laws are now receiving considerable attention (35-38).

Biological distribution systems need to expend energy to overcome viscous losses in the circulatory system, as well as to maintain metabolic processes. To appreciate the underlying biomimetic principles behind the Hess-Murray law, it is informative to consider the power requirements of a biological network. To simplify the analysis, each segment of the transport pathway is considered to be a circular pipe of radius, $r$, and length, $L$. If $Q$ denotes the volumetric flow rate, and $\Delta p$ represents the pressure drop along the segment, then the power required per unit length, $P_{\mathrm{v}}$, to overcome the viscous losses is given by:

$P_{\mathrm{v}}=(\Delta p / L) Q$

[Equation 1]

The volumetric flow rate for fully-developed laminar flow in a pipe can be found from the Hagen-Poiseuille formula (39): 
Figure 2: A schematic representation of a symmetric bifurcating vasculature

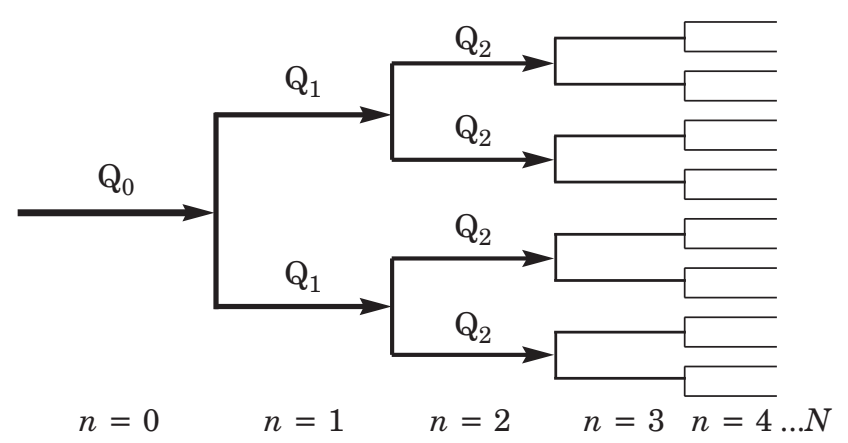

The diagram illustrates the nomenclature used to describe the vascular system, with the inlet channel denoted by $\mathrm{n}=0$ and the finest branches denoted by $\mathrm{n}=\mathrm{N}$. For a symmetrical network, the volumetric flow rate halves at each bifurcation, i.e. $\mathrm{Q}_{n}=2^{-n} \mathrm{Q}_{0}$.

$Q=\frac{\pi r^{4}}{8 \mu} \frac{\Delta p}{L}$

[Equation 2]

where $\mu$ is the coefficient of dynamic viscosity, which is a measure of the fluid's resistance to flow. Substituting $\Delta p / L$ from Equation 2 into Equation 1 allows the viscous power requirement to be written as:

$$
P_{\mathrm{v}}=\frac{8 \mu Q^{2}}{\pi r^{4}}
$$

[Equation 3]

Murray assumed that the power associated with the metabolic requirements of a biological system could be related directly to the volume of each segment. The metabolic power per unit length, $P_{\mathrm{m}}$, required to maintain the blood cells and the walls of the vascular system, can thus be written as:

$P_{\mathrm{m}}=k_{\mathrm{m}} \pi r^{2}$

[Equation 4]

where $k_{\mathrm{m}}$ is a metabolic constant. Since Murray was dealing with cardiovascular systems, he referred to this power requirement as the 'cost of blood volume'. The total power required to operate the system can therefore be written as:

$P=P_{\mathrm{v}}+P_{\mathrm{m}}=\frac{8 \mu Q^{2}}{\pi r^{4}}+k_{m} \pi r^{2}$

[Equation 5]

For a given flow rate, $Q$, the optimum vessel dimension which minimises the power, can be found by differentiating Equation 5 with respect to the radius and equating the resultant expression to zero, i.e.

$\frac{d P}{d r}=-\frac{32 \mu Q^{2}}{\pi r^{5}}+2 \pi k_{\mathrm{m}} r=0$

[Equation 6]
It is now more convenient to work in terms of the diameter of the vessel, $d$. At the optimum conditions, it can readily be shown that the volumetric flow rate can be expressed as:

$Q=\frac{\pi}{32} \sqrt{\frac{k_{m}}{\mu}} d_{o p t}^{3}=M d_{o p t}^{3}$

[Equation 7]

where $M=\pi / 32 \sqrt{\left(k_{\mathrm{m}} / \mu\right)}$ is a constant. The optimum relationship between the diameter of the parent $\left(d_{0}\right)$ and daughter branches $\left(d_{1 \mathrm{a}}\right.$ and $\left.d_{1 \mathrm{~b}}\right)$ can be derived from Equation 7 by using the principle of continuity at the bifurcation, i.e.

$Q_{0}=M d_{0}^{3}=Q_{1 \mathrm{a}}+Q_{1 \mathrm{~b}}=M d_{1 \mathrm{a}}^{3}+M d_{1 \mathrm{~b}}^{3}$

[Equation 8]

which leads to the following relationship:

$d_{0}^{3}=d_{1 \mathrm{a}}^{3}+d_{1 \mathrm{~b}}^{3}$

[Equation 9]

This is now known as the Hess-Murray law, but it is sometimes referred to as the third power law. For a symmetric bifurcation, where $d_{1 \mathrm{a}}=d_{1 \mathrm{~b}}$, it follows that $d_{0}^{3}=2 d_{1}^{3}$. It is informative to consider how the total power requirement shown in Equation 5 varies with the cross-sectional area, $A$. This is illustrated in Figure 3, which shows that the viscous power is inversely proportional to the square of the cross-sectional area, whereas the metabolic power is related linearly to the area. Combined, these two effects give a total power requirement that is a minimum at the optimum vessel size, $A_{\text {opt }}$.

\section{Generalisation of the Hess-Murray Law}

It has recently been shown $(40,41)$ that the Hess-Murray law can be generalised by introducing a branching parameter, $X$, to modify the rate at which the channel dimensions diminish in size. For convenience, the branching parameter can be defined as:

$X=\frac{d_{0}^{3}}{2 d_{1}^{3}}$

[Equation 10]

For $X=1$, the parent/daughter branches obey the principle of minimum work, as defined in Murray's original work. However, the branching parameter does not have to be unity, and the generalised case of $X \neq 1$ can be used to design micro-vasculatures with specific shear stress distributions or residence times. For example, a low shear stress environment will minimise damage to shear-sensitive cells (42), and will increase the probability of cells binding to surfaces (43), whilst increasing the residence time of the fluid in the finest branches of the vasculature will enhance the diffusion of nutrients and waste products.

Assuming that the branching parameter is held constant at each bifurcation, then the diameter of 
Figure 3: Power requirement of a biological fluid distribution system

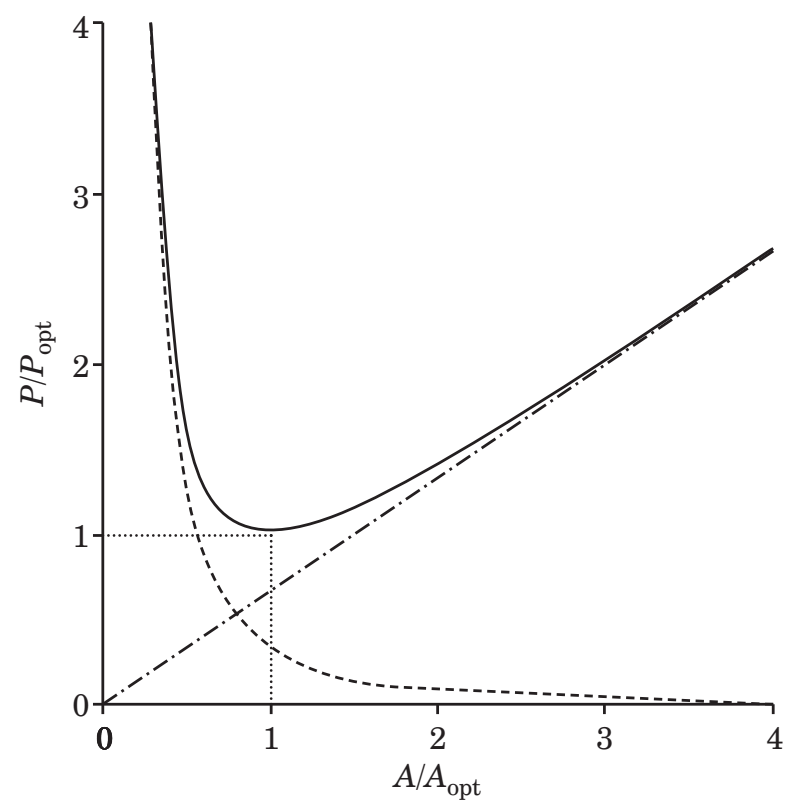

-----= viscous power requirement $\left(\mathrm{P}_{v}\right)$;

$-\cdot-\cdot--=$ metabolic power requirement $\left(\mathrm{P}_{m}\right)$;

$=$ total power requirement $(\mathrm{P})$;

.............. = optimum

A schematic diagram of the power requirement to transport fluid through vessels of varying crosssectional area, $A$. The power required to overcome the viscous losses $\left(\mathrm{P}_{v}\right)$ decreases with the square of the cross-sectional area, whereas the metabolic power requirement to maintain the system $\left(\mathrm{P}_{m}\right)$ is directly proportional to the size of the vessel. The combination of these two effects results in a total power requirement $(\mathrm{P})$ that is a minimum at the optimum vessel size. Adapted from Hess (30) and Weibel (31).

the $n$th generation can be written as:

$d_{n}=\frac{d_{0}}{(2 X)^{n / 3}}$

[Equation 11]

In the case of the Hess-Murray law, $(X=1)$, the diameter of successive generations is reduced by a factor of $2^{-1 / 3}=0.7937$ at each bifurcation, implying that the diameter of the vessels in the vasculature will halve after three successive generations. For a symmetric system, the volumetric flow rate halves at each bifurcation i.e. $Q_{n}=2^{-n} Q_{0}$, so the mean flow velocity, $\bar{U}_{n}$, can be given by:

$\bar{U}_{n}=\bar{U}_{0}\left[\frac{1}{2} X^{2}\right]^{n / 3}$

[Equation 12]

The tangential shear stress acting on the wall of a circular pipe, in a fully-developed laminar flow, is $\tau=8 \mu \bar{U} / d$, so substituting Equations 11 and 12 into the shear stress formula gives: $\tau_{n}=\tau_{0} X^{n}$

[Equation 13]

where $\tau_{0}$ is the wall shear stress in the inlet channel $(n=0)$. Equation 13 clearly indicates an important consequence of the Hess-Murray law $(X=1)$, i.e. that the tangential shear stress at the wall remains constant throughout the vascular network. This is important in tissue-engineering applications, since the shear stress on the cells needs to replicate the precise in vivo cellular conditions found in the organs in vivo. For example, it has been demonstrated that increased levels of wall shear stress stimulate the release of nitric oxide by endothelial cells, which, in turn, inhibits monocyte adhesion to the vessel wall (44). It is therefore vital that the wall shear stress in an artificial vasculature replicates the physiological conditions found in nature.

\section{Vascular Resistance and Residence Time}

To design an effective artificial micro-vasculature for tissue-engineering applications, it is important to ensure that the resistance of the network is compatible with the desired operating pressures and flow rates. It is therefore necessary to be able to estimate the total resistance of the vasculature. For ease of analysis, the derivation is restricted to vascular systems where the lengths of the individual segments are directly proportional to their diameter, as is frequently observed in biological systems (see, for example, West [45]). The hydraulic resistance, $R_{n}=\Delta p / Q$, of an individual segment within the network can be shown to obey $R_{n} \propto d_{n}^{-3}$, so the resistance of a single vessel in the $n$th generation is:

$R_{n}=R_{0}(2 X)^{n}$

[Equation 14]

The total resistance, $R_{\mathrm{T}}$, of a bifurcating vasculature having $N$ generations can be obtained by using the analogy between the pressure differences in pipe flow and the voltage drop in electrical circuits (46). Use of the fact that the individual segments within a given generation are in parallel, gives:

$R_{\mathrm{T}}=R_{0}+\frac{R_{1}}{2}+\frac{R_{2}}{4}+\frac{R_{3}}{8}+\ldots+\frac{R_{N}}{2^{N}} \quad$ [Equation 15]

which can be rewritten as:

$R_{\mathrm{T}}=R_{0}\left[1+X+X^{2}+\ldots+X^{N}\right]=R_{0} \sum_{i=0}^{N} X^{i}$

[Equation 16]

When $X=1$, the resistance of each generation is identical, and the total resistance increases linearly with the number of generations. In this case, Equation 16 reduces to $R_{\mathrm{T}}=(N+1) R_{0}$. If the length of an individual segment is again consid- 
ered to be proportional to its diameter (i.e. $L_{n}=k d_{n}$ where $k$ is a constant), then the average residence time for the $n$th generation can be found from

$t_{n}=\frac{L_{n}}{\bar{U}_{n}}=\frac{k d_{n}}{\bar{U}_{n}}=\frac{k d_{0}}{\bar{U}_{0} X^{n}}=\frac{L_{0}}{\bar{U}_{0} X^{n}}=\frac{t_{0}}{X^{n}}$

[Equation 17]

and the total residence time of the biofluid within the vasculature, $T_{\text {res }}$, can be written as

$T_{\text {res }}=t_{0} \sum_{i=0}^{N} \frac{1}{X^{i}}$

[Equation 18]

By changing the value of $X$, it is possible to introduce an element of control into the residence time of the network. For $X>1$, the residence time decreases at each successive generation. Conversely, for $X<1$, the time to flow through each successive level of the network increases with the number of generations. It should be noted that the resistance and residence time formulae, demonstrated by Equations 16 and 18, are applicable to vasculatures that end at the finest branches, as shown schematically in Figure 2. However, most artificial vasculatures used in tissue-engineering applications are composed of an upstream network of channels to distribute the fluid, together with a downstream network of channels to combine the flow back into a common outlet, as shown in Figure 1b. In this case, it would be necessary to apply a factor of two to Equations 16 and 18. The overall flow resistance of the vasculature, $R_{\mathrm{T}}$, can then be used to estimate the total flow rate through the device, $Q_{0}$. This flow rate is obtained from the following relationship, $Q_{0}=\Delta p / R_{\mathrm{T}}$, where $\Delta p$ is the applied pressure difference across the vasculature. In addition, knowledge of the flow rate then allows the wall shear stress to be determined, to ensure that it lies in the correct physiological range (22).

\section{Extension to Non-circular Ducts}

The extension of the Hess-Murray law to non-circular ducts was first demonstrated by Emerson et al. (40), who proposed that the channels in the vasculature should be designed in accordance with the biomimetic shear stress principle encapsulated in Equation 13. In the interests of brevity, only the final 'design equations' are presented here. A detailed description of the mathematical analysis has been provided by Barber \& Emerson (41).

For a vasculature composed of constant-depth rectangular channels, the biomimetic shear stress principle leads to the following design equation for the optimum aspect ratio of the channels:

$\alpha_{n}\left(1+\alpha_{n}\right) \mathrm{Po}_{n}=(2 X)^{n} \alpha_{0}\left(1+\alpha_{0}\right) \mathrm{Po}_{0}$

[Equation 19]

where $\alpha_{n}=d / w_{n}$ is the channel aspect-ratio (i.e. the ratio of the depth, $d$, to the width, $w_{n}$ ) of the $n$th generation, and $\mathrm{Po}$ is the Poiseuille number which is also a function of the aspect ratio. Equation 19 ensures that the average shear stress around the perimeter of each channel follows the same distribution as that presented in Equation 13. For a rectangular cross-section, the Poiseuille number can be determined analytically, as detailed by Shah \& London (47):

$$
\operatorname{Po}\left(\alpha_{n}^{*}\right)=\frac{24}{\left[1-\frac{192}{\pi^{5}} \frac{1}{\alpha_{n}^{*}} \sum_{i=1,3, \ldots}^{\infty} \frac{1}{i^{5}} \tanh \left(\frac{i \pi \alpha_{n}^{*}}{2}\right)\right]\left(1+\frac{1}{\alpha_{n}^{*}}\right)^{2}}
$$

[Equation 20]

where $\quad \alpha_{n}^{*}=\alpha_{n}$ for $\alpha_{n} \leq 1$

and $\quad \alpha_{n}^{*}=1 / \alpha_{n}$ for $\alpha_{n}>1$.

The channel dimensions in the vascular tree can be found by solving Equation 19 iteratively for $\alpha_{n}$, starting from a known aspect ratio, $\alpha_{0}$, at the inlet of the vasculature. Alternatively, the biomimetic design process can be 'inverted' so that the dimensions of the channels can be expressed in terms of the aspect ratio of the finest channels within the vasculature, $\alpha_{N}$, where $N$ is the total number of generations in the branching network. It can also be shown (see reference 41) that the total resistance of a vasculature composed of constant-depth rectangular channels is the same as that previously presented in Equation 16, provided the length of each

\section{Figure 4: A schematic representation of a symmetric vasculature with a branching index, $m=4$}

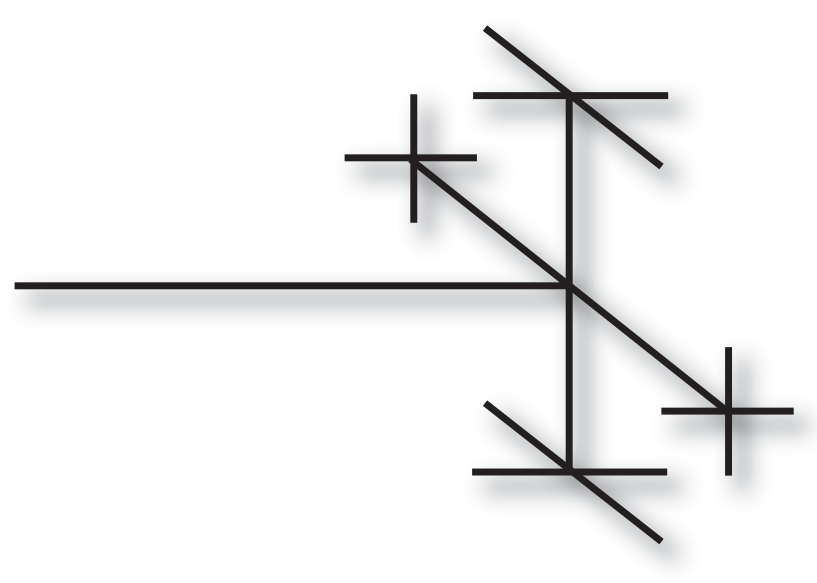

The use of a branching index $\mathrm{m}>2$ may be particularly useful in tissue-engineering applications to increase the number of individual flow channels in the vasculature at a faster rate than a simple bifurcating system. In a $3-D$ scaffold, a branching index of $\mathrm{m}=4$ provides $a$ symmetrical distribution of micro-channels. In this case, the orthogonality of the scaffold offers a simple structure that can readily be fabricated by using existing micro-technologies. 
Figure 5: Wall shear stress distribution in vasculatures that obey the Hess-Murray law

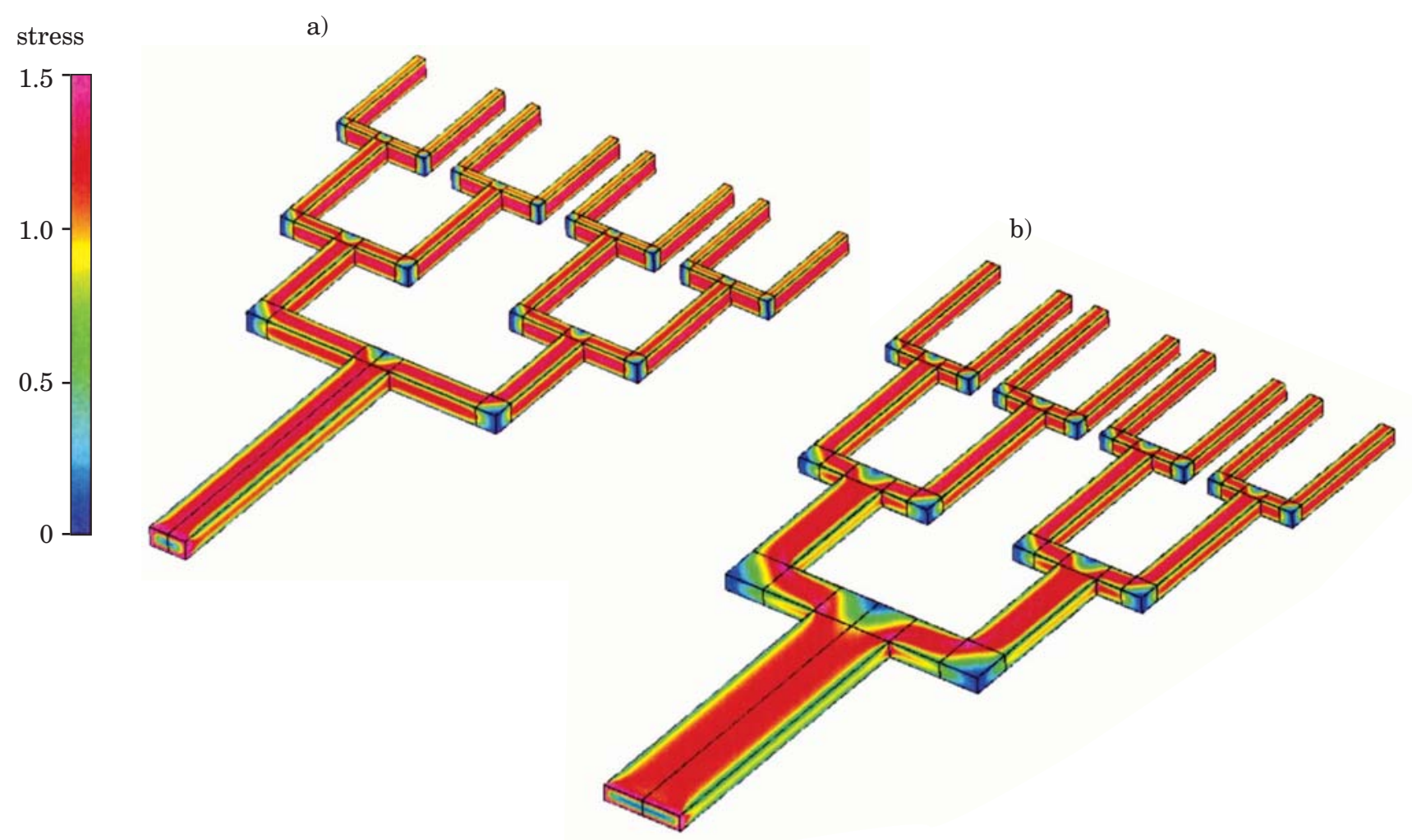

Predicted normalised wall shear stress distributions $\left(\tau / \bar{\tau}_{0}\right)$ in constant-depth rectangular vasculatures that obey the Hess-Murray law (i.e. X = 1). a) Aspect ratio of initial channel, $\alpha_{0}=0.5 ; b$ ) aspect ratio of initial channel, $\alpha_{0}=0.2$. The figure shows that for $\mathrm{X}=1$, the wall shear stress remains constant throughout the vascular tree, as found in arterial/venous systemic flows.

channel is proportional to the hydraulic diameter of the cross-section. However, it should be noted that the residence time of the network does not obey Equation 18. This can be explained by the fact that it is only possible to satisfy a constant average shear stress and a constant residence time, if the Poiseuille number is fixed throughout the system (as found in vasculatures composed of circular pipes). If the Poiseuille number varies at each generation, then it is impossible to simultaneously create a constant shear stress and a constant residence time throughout the vasculature. In practice, it would therefore be necessary to choose the most appropriate design rule for the specific application. For example, a shear stress biomimetic rule would probably be more appropriate for growing engineered tissues, but there may be other applications (e.g. acquisition of biokinetic data), where it is desirable to use a biomimetic rule based on the residence time of the biofluid.

There is no specific requirement in Murray's derivation for the vascular system to bifurcate at each generation, and it can easily be shown that the biomimetic shear stress rule can be applied to any number of daughter branches at each junction. If a constant-depth vasculature splits into $m$ equal-sized branches at each junction, then the biomimetic design rule defined in Equation 19 needs to be reformulated in terms of the branching index, $m$ :

$\alpha_{n}\left(1+\alpha_{n}\right) \mathrm{Po}_{n}=(m X)^{n} \alpha_{0}\left(1+\alpha_{0}\right) \mathrm{Po}_{0}$

[Equation 21]

This modification may be particularly appropriate in 3-D scaffolds, where a branching index of $m=4$ (as shown in Figure 4) appears a logical choice for creating a symmetric arrangement of micro-channels. In this case, the orthogonality of the scaffold offers a simple pragmatic structure that can readily be constructed by using existing micro-fabrication technologies.

\section{Numerical Demonstration of the Biomimetic Design Procedure}

To demonstrate the effectiveness of the biomimetic design rules, a series of computational fluid 
Figure 6: The effect of changing the branching parameter, $x$

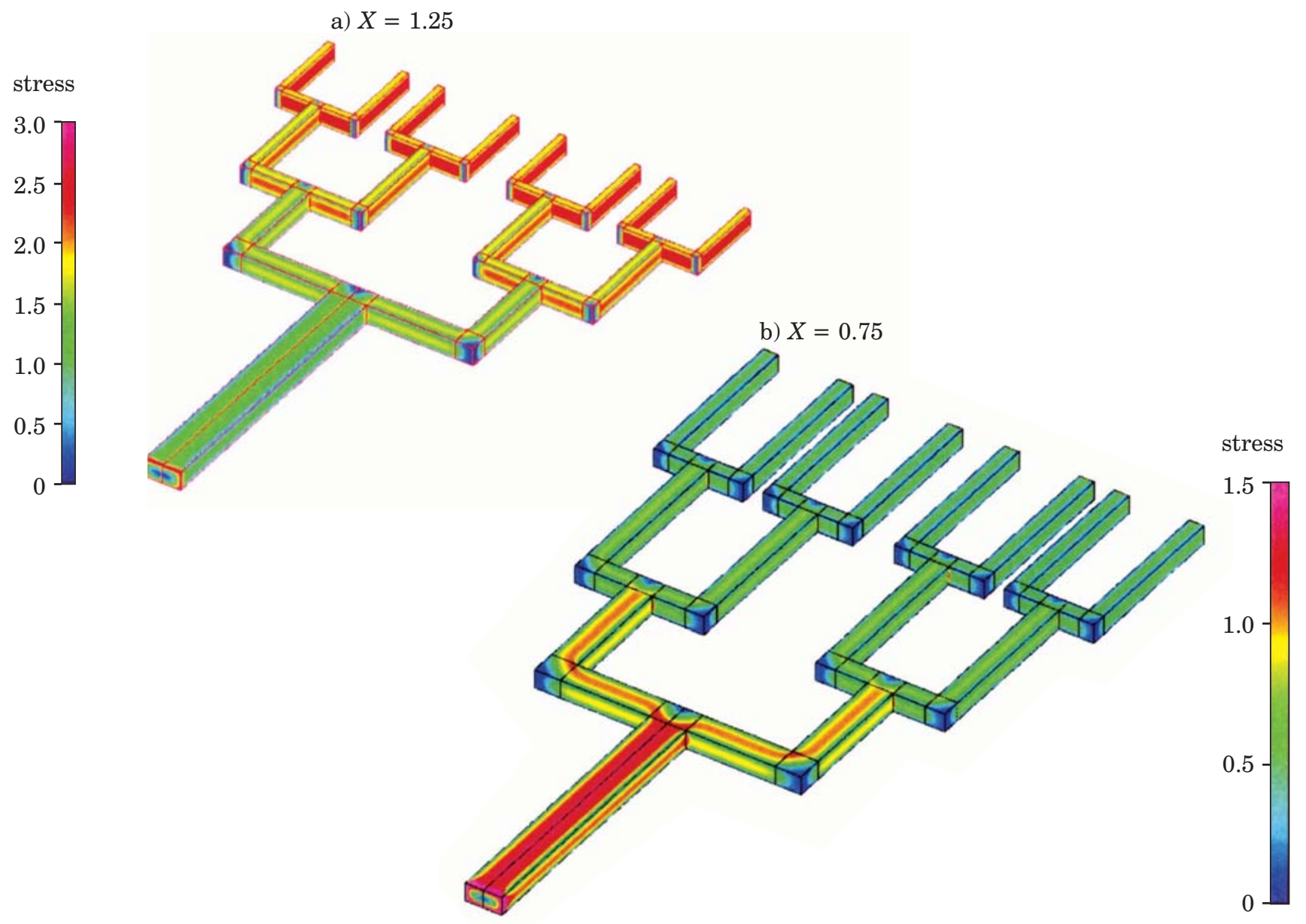

Predicted normalised wall shear stress distributions $\left(\tau / \bar{\tau}_{0}\right)$ in constant-depth rectangular vasculatures, with branching parameters of $\mathrm{X}=1.25$ and $\mathrm{X}=0.75$. The figure clearly shows the progressive increase or decrease in the shear stress as the flow travels toward the finer branches of the vasculature. In this case, the wall shear stress departs from the Hess-Murray law, and the system no longer obeys the principle of minimum work. Decreasing the shear stress toward the finest branches of the vasculature, as shown in b), may be especially useful in tissueengineering applications in order to increase the probability of cells binding to the walls, and also to increase the residence time of the biofluid to enhance the diffusion of nutrients into the tissue culture.

dynamic (CFD) simulations are presented, which highlight the ability to control the shear stress within a vasculature. The first series of simulations considered bifurcating $(m=2)$ constant-depth vasculatures. The networks were restricted to four generations $(n=0,1,2,3)$, and the channels were assumed to have a constant depth of $125 \mu \mathrm{m}$. The initial aspect ratio of the inlet channel was chosen to be either $2: 1\left(\alpha_{0}=0.5\right)$ or $5: 1\left(\alpha_{0}=0.2\right)$. Table 1 shows the channel dimensions obtained by solving Equation 19 for different values of the branching parameter, $X$. Each vasculature also follows the biomimetic principle that the length of each segment is proportional to its hydraulic diameter, $D_{\mathrm{h}}$, which can be defined as $D_{\mathrm{h}}=4 \times$ area/wetted perimeter (39).
The numerical simulations were conducted by using the commercial computational fluid dynamics software package, CFD-ACE+ (48). The meshes representing the networks contained approximately 1.6-2.4 million grid nodes, depending on the geometry and the value of the branching parameter. The simulations assumed that the fluid within the vasculature was pure water, with a dynamic viscosity of $0.001 \mathrm{Ns} / \mathrm{m}^{2}$, and the mean inlet velocity was assumed to be $0.01 \mathrm{~m} / \mathrm{s}$, giving a Reynolds number in the inlet channel, $\operatorname{Re}_{0}=\left(\rho \bar{U}_{0} D_{\mathrm{h}}\right) / \mu$, ranging from 1.66 to 2.08 , depending on the aspect-ratio. Figure 5 shows the predicted normalised wall shear stress distribution, $\tau / \bar{\tau}_{0}$, for vasculatures designed according to 
the Hess-Murray law. It can be seen that for $X=1$, the shear stress remains constant throughout the vascular system. In contrast, Figure 6 illustrates the predicted normalised wall shear stress distributions in vasculatures designed by using branching parameters of $X=1.25$ and $X=0.75$. The figure clearly shows the progressive increase or decrease in the shear stress, as the flow travels towards the finer branches of the network. The effect of varying the branching parameter is further demonstrated in Figure 7, which shows the normalised average shear stress distribution and flow resistance for a range of channel aspect-ratios and branching parameters. The theoretical and numerical predictions are in very good agreement, demonstrating the applicability of the analytical model.

Figure $7 \mathrm{~b}$ graphically demonstrates the effect of varying the value of $X$ on the overall flow resistance of the vasculature. For $X=1$, the resistance of each generation is identical, and the total resistance of the network increases linearly with the number of generations. To achieve this in a network of circular pipes, the diameters of successive generations would need to be reduced by a factor of $2^{-1 / 3}(=0.7937)$ at each bifurcation. However, if the branching parameter is increased to $X=1.25$, which corresponds to a scaling factor of $(2 \times 1.25)^{-1 / 3}(=0.7368)$, then the overall resistance of the vasculature increases rapidly with the number of generations. Thus, relatively small changes in the rate at which the channel dimensions are reduced in size will lead to large variations in the overall flow resistance; this effect is more pronounced when the network contains a large number of generations. It is clear that employing rigorous biomimetic scaling laws to optimise the dimensions of the channels will be of direct benefit to tissue-engineering applications, by ensuring that the total flow resistance of the vasculature is compatible with the desired operating pressures and flow rates.

A second validation study has been carried out to demonstrate the hydrodynamic design of a 3-D scaffold. The investigation considered a constantdepth vasculature with a branching index, $m=4$, as illustrated schematically in Figure 4. Due to the increased number of channels, in comparison to a bifurcating system, the CFD simulations were restricted to three generations $(n=0,1,2)$. The aspect ratio of the inlet channel was taken to be $5: 1$ (i.e. $\alpha_{0}=0.2$ ), and the channels were assumed to have a constant depth of $125 \mu \mathrm{m}$. Table 2 presents

Figure 7: Wall shear stress and flow resistance characteristics
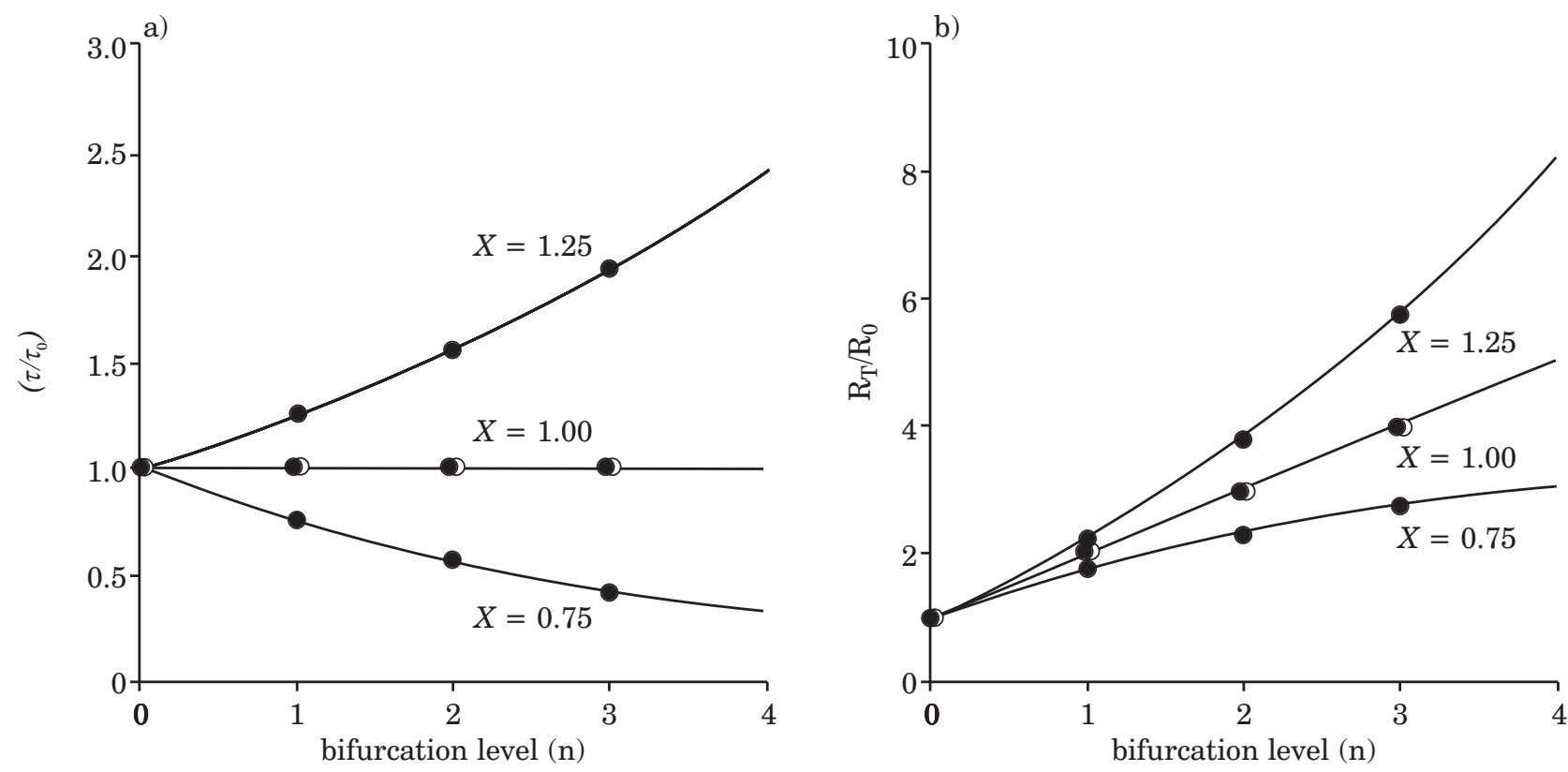

- $=$ CFD results $\left(\alpha_{0}=0.5\right) ; O=C F D$ results $\left(\alpha_{0}=0.2\right) ;-=$ theoretical results.

Normalised wall shear stress distribution a) and flow resistance b) in constant-depth rectangular vasculatures that obey the generalised form of the Hess-Murray law. Comparison is shown between theoretical predictions (lines) and CFD results (symbols). By changing the branching parameter, $\mathrm{X}$, which controls the rate at which the channels diminish in size, it is possible to introduce precise control of the wall shear stress, $\tau$, and the total flow resistance, $\mathrm{R}_{T}$, of the vasculature. 
Table 1: Channel dimensions of constantdepth bifurcating microvasculatures considered in the validation study

\begin{tabular}{lrrrr}
\hline & \multicolumn{4}{c}{ Channel widths $(\boldsymbol{\mu m})$} \\
\cline { 2 - 5 } $\begin{array}{l}\text { Bifurcation } \\
\text { level, } \boldsymbol{n}\end{array}$ & $\boldsymbol{X}=\mathbf{0 . 7 5}$ & $\boldsymbol{X}=\mathbf{1 . 0}$ & $\boldsymbol{X}=\mathbf{1 . 2 5}$ & $\boldsymbol{X}=\mathbf{1 . 0}$ \\
\hline 0 & 250.0 & 250.0 & 250.0 & 625.0 \\
1 & 177.7 & 143.3 & 123.0 & 312.9 \\
2 & 132.0 & 91.8 & 71.4 & 171.5 \\
3 & 101.7 & 62.5 & 44.2 & 106.3 \\
\hline
\end{tabular}

The table presents the channel dimensions of a series of constant-depth rectangular-sectioned vasculatures employed in the numerical validation study. Following Lim et al. (28), the channels are assumed to have a constant depth of $125 \mu \mathrm{m}$ and the initial aspect-ratio of the inlet channel was chosen to be either 2:1 $\left(\alpha_{0}=0.5\right)$ or $5: 1\left(\alpha_{0}=0.2\right)$. The channel dimensions are obtained by solving Equation 19 for the aspect-ratio, $\alpha_{n}$, and then computing the channel width from $w_{n}=d / \alpha_{n}$. Each vasculature also follows the biomimetic principle that the length of each segment is proportional to its hydraulic diameter, $\mathrm{D}_{h}$. Representative values of the branching parameter ranging from $\mathrm{X}=0.75$ to $\mathrm{X}=1.25$ were chosen for the present study. the channel dimensions obtained by solving Equation 21. The computational mesh used to represent the vasculature contained approximately 1.4-million grid nodes, and again, the vasculature used the biomimetic principle that the length of each segment is proportional to its hydraulic diameter.

Figure 8 illustrates the predicted normalised shear stress distribution along the walls of the scaffold. It can be seen that the shear stress remains identical between successive generations. A detailed examination of the shear stress profiles shows that the tangential shear stress around the perimeter of each channel varies slightly with generation level, $n$. This is inevitable, since the aspect ratios of the channels vary at each generation. However, as shown in Table 3, the average wall shear stress around the perimeter remains constant throughout the vasculature, indicating that the biomimetic design rule presented in Equation 21 can be applied to networks with an arbitrary splitting index, $m$. The numerical validation studies have therefore demonstrated that it is possible to optimise the channel dimensions within artificial 2-D and 3-D micro-vasculatures, to permit precise control of the wall shear stress distribution or total flow resistance of the network. This is important in tissue-engineering applications, so that the hydrodynamics of the vasculature replicate the exact in vivo conditions found in natural tissues.

\section{Figure 8: Wall shear stress distribution in a 3-D vasculature that obeys the Hess-Murray law}
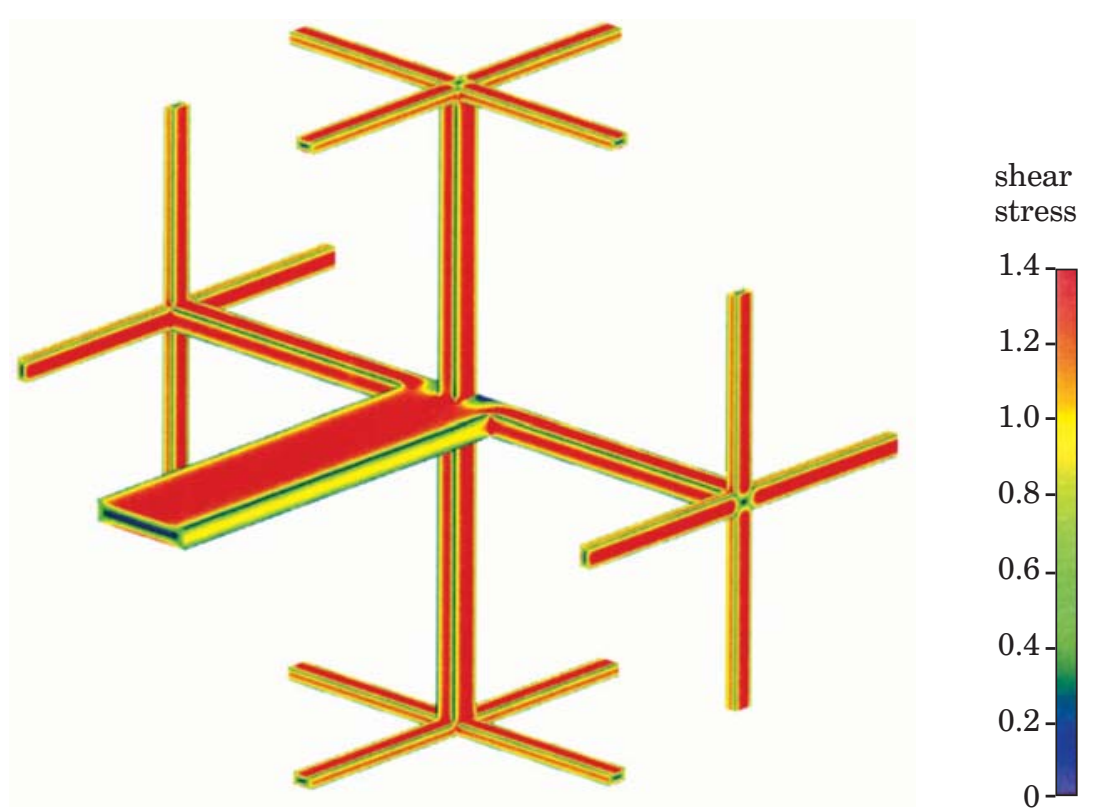

Predicted normalised wall shear stress distribution $\left(\tau / \bar{\tau}_{0}\right)$ in a $3-D$ scaffold with $\mathrm{m}=4$ and $\mathrm{X}=1$. The figure shows that, for $\mathrm{X}=1$, the wall shear stress remains constant throughout the vascular tree, thereby replicating the conditions found in arterial/venous systemic flows. 
Table 2: Channel dimensions in a constantdepth 3-D micro-vasculature

\begin{tabular}{lccc}
\hline Level, & $\begin{array}{c}\text { Aspect } \\
\text { ratio, } \\
\boldsymbol{a}_{\boldsymbol{n}}\end{array}$ & $\begin{array}{c}\text { Channel } \\
\text { width, } \\
\boldsymbol{w}_{\boldsymbol{n}}(\boldsymbol{\mu} \mathbf{m})\end{array}$ & $\begin{array}{c}\text { Hydraulic } \\
\text { diameter, } \\
\boldsymbol{D}_{\boldsymbol{h}}(\boldsymbol{\mu} \mathrm{m})\end{array}$ \\
\hline 0 & 0.200 & 625.00 & 208.3 \\
1 & 0.729 & 171.48 & 144.6 \\
2 & 1.756 & 71.18 & 90.7 \\
\hline
\end{tabular}

The table presents the optimised channel dimensions for a constant-depth 3-D micro-vasculature having a branching index, $\mathrm{m}=4$ (i.e. the vasculature splits into 4 branches at each junction). For consistency with the previous validation study, the channels are assumed to have a constant depth of $125 \mu \mathrm{m}$ and the initial aspect ratio of the inlet channel was chosen to be 5:1 $\left(\alpha_{0}=0.2\right)$. In the case of a 3-D vasculature, the optimised channel dimensions are obtained by solving Equation 21 for the aspect ratio, $\alpha_{n}$, and then computing the channel width from $w_{n}=d / \alpha_{n}$. The vasculature again follows the biomimetic principle that the length of each segment is proportional to its hydraulic diameter.

\section{Conclusions}

This article has shown how Murray's law, which governs the optimum ratio between the diameters of the parent and daughter branches in biological fluid distribution networks, can be used to design the artificial vascularised constructs found in tissue-engineering applications. Murray's law was originally developed for cardiovascular systems composed of multi-diameter circular pipes, and uses the principle of minimum work to derive the optimum rate at which the vessels in the network diminish in size. An important consequence of Murray's law is that the tangential shear stress at the wall remains constant throughout the vasculature. This important biomimetic principle can be generalised, so that it can be applied to fluid distribution networks composed of channels of arbitrary cross-section.

This article considers the design of constantdepth, rectangular-sectioned vasculatures that are often employed in lab-on-a-chip systems. By introducing a branching parameter that governs the rate at which the channels diminish in size, it is shown that it is possible to introduce precise control over the shear stress and residence time in a hierarchical network. This may be particularly useful in tissue-engineering applications, in order to assist the adhesion of cells to the channel walls, and to enhance the diffusion of nutrients and waste products into and from the tissue culture. The use of rigorous biomimetic scaling rules to optimise the channel dimensions within an artificial vasculature will clearly be of direct benefit in the development of the next generation of vascularised tissue constructs.
Table 3: Predicted mean wall shear stress in a constant-depth 3-D microvasculature

\begin{tabular}{lcc} 
Level, $\boldsymbol{n}$ & $\begin{array}{c}\text { Mean wall } \\
\text { shear stress }\left(\mathbf{N} / \mathbf{m}^{\mathbf{2}}\right)\end{array}$ & $\begin{array}{c}\text { Normalised mean } \\
\text { wall shear stress }\end{array}$ \\
\hline 0 & 0.439 & 1.000 \\
1 & 0.436 & 0.993 \\
2 & 0.434 & 0.989 \\
\hline
\end{tabular}

Predicted normalised wall shear stress distribution $\left(\tau / \bar{\tau}_{0}\right)$ in a 3-D scaffold with $\mathrm{m}=4$ and $\mathrm{X}=1$. The table shows that, for $\mathrm{X}=1$, the wall shear stress remains constant throughout the vascular tree, replicating the conditions found in arterial/venous systemic flows.

\section{Acknowledgements}

The authors would like to acknowledge the support provided by the UK Engineering and Physical Sciences Research Council (EPSRC) under the auspices of Collaborative Computational Project 12 (CCP12). In addition, the authors would like to thank Professor Krzysztof Cieślicki of Warsaw University of Technology for the many useful discussions on Murray's law and Dr Nirmala Bhogal of FRAME for her detailed comments on the manuscript.

\section{References}

1. Langer, R. \& Vacanti, J.P. (1993). Tissue engineering. Science, New York 260, 920-926.

2. Griffith, L.G., Wu, B., Cima, M.J., Powers, M.J., Chaignaud, B. \& Vacanti, J.P. (1997). In vitro organogenesis of liver tissue. Annals of the New York Academy of Sciences 831, 382-397.

3. Bhatia, S.N. \& Chen, C.S. (1999). Tissue engineering at the micro-scale. Biomedical Microdevices 2, 131-144.

4. Desai, T.A. (2000). Micro- and nanoscale structures for tissue engineering constructs. Medical Engineering \& Physics 22, 595-606.

5. Kaihara, S., Borenstein, J., Koka, R., Lalan, S., Ochoa, E.R., Ravens, M., Pien, H., Cunningham, B. \& Vacanti, J.P. (2000). Silicon micromachining to tissue engineer branched vascular channels for liver fabrication. Tissue Engineering 6, 105-117.

6. Griffith, L.G. \& Naughton, G. (2002). Tissue engineering - Current challenges and expanding opportunities. Science, New York 295, 1009-1014.

7. Borenstein, J.T., Terai, H., King, K.R., Weinberg, E.J., Kaazempur-Mofrad, M.R. \& Vacanti, J.P. (2002). Microfabrication technology for vascularized tissue engineering. Biomedical Microdevices 4, $167-175$.

8. Tsang, V.L. \& Bhatia, S.N. (2004). Three-dimensional tissue fabrication. Advanced Drug Delivery Reviews 56, 1635-1647.

9. Khademhosseini, A., Langer, R., Borenstein, J. \& 
Vacanti, J.P. (2006). Microscale technologies for tissue engineering and biology. Proceedings of the National Academy of Sciences of the USA 103, 2480-2487.

10. Sarkar, S., Lee, G.Y., Wong, J.Y. \& Desai, T.A. (2006). Development and characterization of a porous micro-patterned scaffold for vascular tissue engineering applications. Biomaterials $\mathbf{2 7}$ 4775-4782.

11. Yang, Y. \& El Haj, A.J. (2006). Biodegradable scaffolds - delivery systems for cell therapies. Expert Opinion on Biological Therapy 6, 485-498.

12. Borenstein, J.T., Weinberg, E.J., Orrick, B.K., Sundback, C., Kaazempur-Mofrad, M.R. \& Vacanti, J.P. (2007). Microfabrication of three-dimensional engineered scaffolds. Tissue Engineering 13, 1837-1844.

13. Toh, Y.C., Zhang, C., Zhang, J., Khong, Y.M., Chang, S., Samper, V.D., van Noort, D., Hutmacher, D.W. \& $\mathrm{Yu}, \mathrm{H}$. (2007). A novel 3D mammalian cell perfusionculture system in microfluidic channels. Lab on a Chip 7, 302-309.

14. Hoganson, D.M., Pryor, H.I. \& Vacanti, J.P. (2008). Tissue engineering and organ structure: A vascularized approach to liver and lung. Pediatric Research 63, 520-526.

15. Du, Y., Cropek, D., Kaazempur-Mofrad, M.R., Weinberg, E.J., Khademhosseini, A. \& Borenstein, J. (2008). Microfluidic systems for engineering vascularized tissue constructs. In Microfluidics for Biological Applications (ed. W-C. Tian \& E. Finehout), Chapter 7, pp. 223-240. New York, NY, USA: Springer.

16. Colton, C.K. (1995). Implantable biohybrid artificial organs. Cell Transplantation 4, 415-436.

17. Whitesides, G.M., Ostuni, E., Takayama, S., Jiang, X. \& Ingber, D.E. (2001). Soft lithography in biology and biochemistry. Annual Review of Biomedical Engineering 3, 335-373.

18. King, K.R., Wang, C.C.J., Kaazempur-Mofrad, M.R., Vacanti, J.P. \& Borenstein, J.T. (2004). Biodegradable microfluidics. Advanced Materials 16, 2007-2012.

19. Bettinger, C.J., Weinberg, E.J., Kulig, K.M., Vacanti, J.P., Wang, Y., Borenstein, J.T. \& Langer, R. (2006). Three-dimensional microfluidic tissue-engineering scaffolds using a flexible biodegradable polymer. Advanced Materials 18, 165-169.

20. Cabodi, M., Choi, N.W., Gleghorn, J.P., Lee, C.S.D., Bonassar, L.J. \& Stroock, A.D. (2005). A microfluidic biomaterial. Journal of the American Chemical Society 127, 13,788-13,789.

21. Kaazempur-Mofrad, M.R., Vacanti, J.P., Krebs, N.J. \& Borenstein, J.T. (2004). A MEMS-based renal replacement system. Proceedings of the 2004 Solid-state Sensor, Actuator and Microsystems Workshop, 6-10 June 2004, pp. 67-70. San Diego, CA, USA: Transducer Research Foundation.

22. Sin, A., Baxter, G.T. \& Shuler, M.L. (2001). Animal on a chip: A microscale cell culture analog device for evaluating toxicological and pharmacological profiles. In Microfluidics and BioMEMS, Proceedings of SPIE, Volume 4560, pp. 98-101 (ed. C.H. Mastrangelo \& H. Becker). Bellingham, WA, USA: SPIE.

23. Khamsi, R. (2005). Meet the stripped down rat. Nature, London 435, 12-13.

24. Viravaidya, K., Sin, A. \& Shuler, M.L. (2004). Development of a microscale cell culture analog to probe naphthalene toxicity. Biotechnology Progress 20, 316-323.

25. Wang, Y., Ameer, G.A., Sheppard, B.J. \& Langer, R. (2002). A tough biodegradable elastomer. Nature Biotechnology 20, 602-606.

26. Weaver, V.M., Petersen, O.W., Wang, F., Larabell, C.A., Briand, P., Damsky, C. \& Bissell, M.J. (1997). Reversion of the malignant phenotype of human breast cells in three-dimensional culture and in vivo by integrin blocking antibodies. Journal of Cell Biology 137, 231-245.

27. Therriault, D., White, S.R. \& Lewis, J.A. (2003). Chaotic mixing in three-dimensional microvascular networks fabricated by direct-write assembly. Nature Materials 2, 265-271.

28. Lim, D., Kamotani, Y., Cho, B., Mazumder, J. \& Takayama, S. (2003). Fabrication of microfluidic mixers and artificial vasculatures using a highbrightness diode-pumped Nd:YAG laser direct write method. Lab on a Chip 3, 318-323.

29. Murray, C.D. (1926). The physiological principle of minimum work: I. The vascular system and the cost of blood volume. Proceedings of the National Academy of Sciences of the USA 12, 207-214.

30. Hess, W.R. (1914). Das Prinzip des kleinsten Kraftverbrauches im Dienste hämodynamischer Forschung. Archiv für Anatomie und Physiologie: Physiologische Abtheilung 1/2, 1-59.

31. Weibel, E.R. (2000). Symmorphosis: On Form and Function in Shaping Life, 263pp. Cambridge, MA, USA: Harvard University Press.

32. Hahn, H.K., Georg, M. \& Peitgen, H-O. (2005). Fractal aspects of three-dimensional vascular constructive optimization. In Fractals in Biology and Medicine (ed. G.A. Losa, D. Merlini, T.F. Nonnenmacher \& E.R. Weibel), pp. 55-66. Basel, Switzerland: Birkhäuser.

33. Bejan, A. \& Lorente, S. (2008). Design with Constructal Theory, 529pp. Hoboken, NJ, USA: John Wiley \& Sons.

34. Sherman, T.F. (1981). On connecting large vessels to small: The meaning of Murray's Law. Journal of General Physiology 78, 431-453.

35. West, G.B., Brown, J.H. \& Enquist, B.J. (1997). A general model for the origin of allometric scaling laws in biology. Science, New York 276, 122-126.

36. Zhou, Y., Kassab, G.S. \& Molloi, S. (1999). On the design of the coronary arterial tree: A generalization of Murray's law. Physics in Medicine \& Biology 44, 2929-2945.

37. Banavar, J.R., Maritan, A. \& Rinaldo, A. (1999). Size and form in efficient transportation networks. Nature, London 399, 130-132.

38. West, G.B. (1999). The origin of universal scaling laws in biology. Physica A 263, 104-113.

39. White, F.M. (1991). Viscous Fluid Flow, 2nd edn, 614pp. New York, NY, USA: McGraw-Hill.

40. Emerson, D.R., Cieślicki, K., Gu, X.J. \& Barber, R.W. (2006). Biomimetic design of microfluidic manifolds based on a generalised Murray's law. Lab on a Chip 6, 447-454.

41. Barber, R.W. \& Emerson, D.R. (2008). Optimal design of microfluidic networks using biologically inspired principles. Microfluidics \& Nanofluidics 4, 179-191.

42. Ma, N., Koelling, K.W. \& Chalmers, J.J. (2002). Fabrication and use of a transient contractional flow device to quantify the sensitivity of mammalian and insect cells to hydrodynamic forces. 
Biotechnology \& Bioengineering 80, 428-437.

43. Auninš̌, J.G., Bader, B., Caola, A., Griffiths, J., Katz, M., Licari, P., Ram, K., Ranucci, C.S. \& Zhou, W. (2003). Fluid mechanics, cell distribution, and environment in cellcube bioreactors. Biotechnology Progress 19, 2-8.

44. Tsao, P.S., Lewis, N.P., Alpert, S. \& Cooke, J.P. (1995). Exposure to shear stress alters endothelial adhesiveness - Role of nitric oxide. Circulation 92, 3513-3519.

45. West, B.J. (1990). Fractal Physiology and Chaos in
Medicine, 278pp. Singapore: World Scientific Publishing.

46. Ajdari, A. (2004). Steady flows in networks of microfluidic channels: Building on the analogy with electrical circuits. Comptes Rendus Physique 5, 539546.

47. Shah, R.K. \& London, A.L. (1978). Laminar Flow Forced Convection in Ducts, 477pp. New York, NY, USA: Academic Press.

48. Anon. (2008). CFD-ACE+User Manual - V2008.2, 542pp. Huntsville, AL, USA: ESI CFD, Inc. 